\title{
SYMMETRIC IMPRIMITIVITY THEOREMS FOR GRAPH $C^{*}$-ALGEBRAS
}

\author{
DAVID PASK AND IAIN RAEBURN
}

The $C^{*}$-algebra $C^{*}(E)$ of a directed graph $E$ is generated by partial isometries satisfying relations which reflect the path structure of the graph. In [10], Kumjian and Pask considered the action of a group $G$ on $C^{*}(E)$ induced by an action of $G$ on $E$. They proved that if $G$ acts freely and $E$ is locally finite, then the crossed product $C^{*}(E) \times G$ is Morita equivalent to the $C^{*}$-algebra of the quotient graph $E / G$ [10, Corollary 3.10].

This theorem bears a striking resemblance to a famous theorem of Green, which says that the crossed product $C_{0}(X) \times G$ associated to a free and proper action of $G$ on a locally compact space $X$ is Morita equivalent to $C_{0}(X / G)$ [6]. So one naturally asks whether this resemblance can be pushed further: are there analogues for free actions on graphs of the other Morita equivalences associated to free and proper actions on spaces? Here we contribute to this circle of ideas by proving an analogue of the symmetric imprimitivity theorem of [15] and [12] concerning commuting free and proper actions of two different groups.

The proofs of the Kumjian-Pask theorem in [10] and [9] rely on a result of Gross and Tucker which realises free actions as translations on skew products, and exhibit a stable isomorphism rather than a Morita equivalence. Here choosing a skew-product realisation for one of the actions would destroy the symmetry of the situation, so instead we construct directly an imprimitivity bimodule implementing the equivalence. This construction is of some interest even in the case of one action; it shows, for example, that the actions on $C^{*}(E)$ induced by free actions on $E$ provide a new family of actions which are proper and saturated in the sense of Rieffel ([16], see also $[5,8,17])$. Since our methods do not require any local-finiteness hypothesis on the graph $E$, our one-action result is more general than the original Kumjian-Pask theorem.

In fact we shall prove two symmetric imprimitivity theorems, one for reduced crossed products (Theorem 1.9) and one for full crossed products (Theorem 2.1). We prove the theorem about reduced crossed products in $\S 1$, following the strategy of [4]. We show that every action on $C^{*}(E)$ induced by a free action on $E$ is proper in Rieffel's sense, so that the machine of [16] gives a bimodule for each action. We then take crossed products of these bimodules by actions of the other groups, and tensor them to get a bimodule implementing the desired equivalence. In $\S 2$, we prove

Date: February 15, 2002.

This research was supported by a grant from the University of Newcastle. 
the theorem for full crossed products. This time we build the bimodule directly, as in [15] and [12]; this second construction uses much the same technical tools as we required in $\S 1$, so there is little redundancy in our approach. In the last section we give some applications, and in particular show how comparing the two versions of the imprimitivity theorem can lead to amenablity results (Corollary 3.1).

Conventions. A directed graph $E=\left(E^{0}, E^{1}, r, s\right)$ consists of countable sets $E^{0}$ of vertices and $E^{1}$ of edges, and range and source maps $r, s: E^{1} \rightarrow E^{0}$. For graph algebras, we use the conventions of [13]. Thus a Cuntz-Krieger family $\left\{S_{e}, P_{v}\right\}$ consists of partial isometries $\left\{S_{e}: e \in E^{1}\right\}$ with mutually orthogonal ranges and mutually orthogonal projections $\left\{P_{v}: v \in E^{0}\right\}$ such that

$$
S_{e}^{*} S_{e}=P_{r(e)}, \quad S_{e} S_{e}^{*} \leq P_{s(e)} \quad \text { and } \quad P_{v}=\sum_{s(e)=v} S_{e} S_{e}^{*} \text { whenever } 0<\left|s^{-1}(v)\right|<\infty .
$$

For row-finite graphs, this reduces to the usual definition in [11] or [1], so those interested in finite or row-finite graphs can just ignore the extra generality. The graph $C^{*}$-algebra $C^{*}(E)$ is generated by a universal Cuntz-Krieger family $\left\{s_{e}, p_{v}\right\}$; we write $\pi_{S, P}$ for the representation of $C^{*}(E)$ corresponding to a Cuntz-Krieger family $\left\{S_{e}, P_{v}\right\}$. We write $E^{*}$ for the path space of $E$, and for $\mu \in E^{*}$ of length $|\mu|$, we write $s_{\mu}:=s_{\mu_{1}} \cdots s_{\mu_{|\mu|}}$. As usual, the elements of the form $s_{\mu} s_{\nu}^{*}$ span a dense *-subalgebra of $C^{*}(E)$.

\section{REDUCED CROSSED PRODUCTS}

Let $E$ be a directed graph, and suppose we have a left action of a group $G$ on $E$ which is free on $E^{0}$ (and hence on $E^{1}$ ). By universality of $C^{*}(E)$ there is an induced action $\alpha: G \rightarrow$ Aut $C^{*}(E)$ such that $\alpha_{g}\left(s_{e}\right)=s_{g \cdot e}$ and $\alpha_{g}\left(p_{v}\right)=p_{g \cdot v}$. We aim to show that the action $\alpha$ is proper and saturated in the sense of [16].

Let

$$
X_{0}(E)=\operatorname{span}\left\{s_{\mu} s_{\nu}^{*}: \mu, \nu \in E^{*} \text { and } r(\mu)=r(\nu)\right\},
$$

which is a dense $\alpha$-invariant $*$-subalgebra of $C^{*}(E)$. As in [16], we denote by $M\left(X_{0}(E)\right)$ the set of multipliers of $C^{*}(E)$ which multiply $X_{0}(E)$ into itself.

Lemma 1.1. There is a $*$-linear map $I_{G}: X_{0}(E) \rightarrow M\left(X_{0}(E)\right)^{\alpha}$ such that

$$
x I_{G}(y)=\sum_{g \in G} x \alpha_{g}(y) \text { and } I_{G}(y) x=\sum_{g \in G} \alpha_{g}(y) x \quad \text { for } x, y \in X_{0}(E) .
$$

Proof. We begin by showing that there is a suitable multiplier $I_{G}(y)$ when $y=s_{\mu} s_{\nu}^{*}$. For each generator $s_{\alpha} s_{\beta}^{*}$,

$$
s_{\alpha} s_{\beta}^{*} \alpha_{g}\left(s_{\mu} s_{\nu}^{*}\right)=s_{\alpha} s_{\beta}^{*} s_{g \cdot \mu} s_{g \cdot \nu^{*}}
$$

is nonzero for at most one $g$, namely the one for which $s(\beta)=s(g \cdot \mu)=g$. $s(\mu)$. This implies, first, that the sums in (1.1) are always finite, and, second, that $x\left(\sum_{g \in G} \alpha_{g}(y)\right)=x\left(\sum_{g \in F} \alpha_{g}(y)\right)$ for any large enough finite subset $F$ of $G$ (and similarly on the right). Since the action of $G$ is free, the partial isometries 
$\alpha_{g}\left(s_{\mu} s_{\nu}^{*}\right)$ have mutually orthogonal ranges and domains. Thus it follows from a minor variation of the argument of [1, Lemma 1.1] that the series $\sum_{g \in G} \alpha_{g}\left(s_{\mu} s_{\nu}^{*}\right)$ converges strictly to a partial isometry $I_{G}\left(s_{\mu} s_{\nu}^{*}\right)$ in $M\left(C^{*}(E)\right)$ satisfying

$$
x I_{G}\left(s_{\mu} s_{\nu}^{*}\right)=\sum_{g \in G} x \alpha_{g}\left(s_{\mu} s_{\nu}^{*}\right)
$$

since this sum is finite, the multiplier $I_{G}\left(s_{\mu} s_{\nu}^{*}\right)$ belongs to $M\left(X_{0}(E)\right)$. It is trivial to check that $I_{G}\left(s_{\mu} s_{\nu}^{*}\right)$ is fixed by $\alpha$.

It remains to extend $I_{G}$ to all of $X_{0}(E)=\operatorname{span}\left\{s_{\mu} s_{\nu}^{*}\right\}$. Let $y:=\sum_{\mu, \nu} \lambda_{\mu, \nu} s_{\mu} s_{\nu}^{*}$ be a finite sum in $X_{0}(E)$. Since there is at most one element $I_{G}(y)$ of $M\left(C^{*}(E)\right)$ satisfying (1.1) for all $x$, and $\sum_{\mu, \nu} \lambda_{\mu, \nu} I_{G}\left(s_{\mu} s_{\nu}^{*}\right)$ has this property, we can safely define $I_{G}(y):=\sum_{\mu, \nu} \lambda_{\mu, \nu} I_{G}\left(s_{\mu} s_{\nu}^{*}\right)$. A similar argument shows that $I_{G}$ is then $*$-linear.

Corollary 1.2. The action $\alpha$ of $G$ on $C^{*}(E)$ is proper with respect to the subalgebra $X_{0}(E)$.

Proof. We just need to observe that $g \mapsto x \alpha_{g}(y)$ has finite support, so that it is trivially in $\ell^{1}\left(G, C^{*}(E)\right)$, and take $\langle x, y\rangle_{D}:=I_{G}\left(x^{*} y\right)$.

Next we have to identify the generalised fixed-point algebra $C^{*}(E)^{\alpha}$. To do this, we need more information about the map $I_{G}$ of Lemma 1.1. It is definitely not a homomorphism: we have $s_{e} s_{f}=0$ but $I_{G}\left(s_{e}\right) I_{G}\left(s_{f}\right) \neq 0$ when $r(e)=g \cdot s(f)$ for some $g$ which is not the identity element of $G$. However, if $s_{e} s_{f} \neq 0$, so that $e f$ is a path in $E$, then $I_{G}$ behaves nicely:

Lemma 1.3. If $\mu, \nu \in E^{*}$ satisfy $r(\mu)=r(\nu)$, so that $s_{\mu} s_{\nu}^{*} \neq 0$ in $C^{*}(E)$, then

$$
I_{G}\left(s_{\mu} s_{\nu}^{*}\right)=I_{G}\left(s_{\mu_{1}}\right) I_{G}\left(s_{\mu_{2}}\right) \cdots I_{G}\left(s_{\mu_{|\mu|}}\right) I_{G}\left(s_{\nu_{|\nu|}}\right)^{*} \cdots I_{G}\left(s_{\nu_{1}}\right)^{*} .
$$

Proof. First suppose that $r(e)=s(f)$. Then by freeness we have

$$
r(g \cdot e)=s(k \cdot f) \Longrightarrow g \cdot r(e)=k \cdot s(f) \Longrightarrow g=k,
$$

and so $s_{g \cdot e} s_{k \cdot f} \neq 0$ only when $g=k$. Thus

$$
I_{G}\left(s_{e}\right) I_{G}\left(s_{f}\right) x=\sum_{g, k \in G} s_{g \cdot e} s_{k \cdot f} x=\sum_{g \in G} s_{g \cdot e} s_{g \cdot f} x=I_{G}\left(s_{e} s_{f}\right) x .
$$

An induction argument gives the result for arbitrary $\mu$ and $\nu=\emptyset$. Now note that $s_{\mu} s_{\nu}^{*} \neq 0$ iff $r(\mu)=r(\nu)$, and the same argument gives $I_{G}\left(s_{\mu}\right) I_{G}\left(s_{\nu}\right)^{*}=I_{G}\left(s_{\mu} s_{\nu}^{*}\right)$.

Every path $\mu$ in $E$ gives a path in the quotient graph, which we denote by $G \cdot \mu$. In fact every path in $G \backslash E$ has the form $G \cdot \mu$ : if $\alpha=\left(G \cdot e_{1}\right)\left(G \cdot e_{2}\right) \cdots$ is a path in $G \backslash E$, then we can find $g_{i} \in G$ such that $\mu=e_{1}\left(g_{2} \cdot e_{2}\right)\left(g_{3} \cdot e_{3}\right) \cdots$ is a path in $E$, and then $\alpha=G \cdot \mu$. Thus we have

$$
C^{*}(G \backslash E)=\overline{\operatorname{span}}\left\{s_{G \cdot \mu} s_{G \cdot \nu}: \mu, \nu \in E^{*}\right\} .
$$


Lemma 1.4. The elements $T_{G \cdot e}:=I_{G}\left(s_{e}\right), Q_{G \cdot v}:=I_{G}\left(p_{v}\right)$ form a Cuntz-Krieger $G \backslash E$-family in $M\left(C^{*}(E)\right)$. Thus there is a nondegenerate homomorphism $\phi_{G}$ : $C^{*}(G \backslash E) \rightarrow M\left(C^{*}(E)\right)$ such that

$$
\phi_{G}\left(s_{G \cdot \mu} s_{G \cdot \nu}^{*}\right)=I_{G}\left(s_{\mu} s_{\nu}^{*}\right) \text { for all } \mu, \nu \in E^{*},
$$

and $\phi_{G}$ is an isomorphism of $C^{*}(G \backslash E)$ onto the $C^{*}$-subalgebra of $M\left(C^{*}(E)\right)$ generated by $\left\{T_{G \cdot e}, Q_{G \cdot v}\right\}$.

Proof. We have already observed in the proof of Lemma 1.1 that $I_{G}\left(s_{e}\right)$ is a partial isometry, and it follows immediately from [1, Lemma 1.1] that $I_{G}\left(p_{v}\right)$ is a projection. For the first Cuntz-Krieger relation, we use freeness again:

$$
T_{G \cdot e}^{*} T_{G \cdot e} x=\sum_{g \in G} \sum_{k \in G} s_{g \cdot e}^{*} s_{k \cdot e} x=\sum_{g \in G} s_{g \cdot e}^{*} s_{g \cdot e} x=I_{G}\left(p_{r(e)}\right) x=Q_{r(G \cdot e)} x .
$$

Next suppose $G \cdot e \neq G \cdot f$. Then the partial isometries $\left\{s_{g \cdot e}, s_{k \cdot f}: g, k \in G\right\}$ have orthogonal ranges, and hence

$$
\left(T_{G \cdot e} T_{G \cdot e}^{*}\right)\left(T_{G \cdot f} T_{G \cdot f}^{*}\right) x=\sum_{g, k \in G}\left(s_{g \cdot e} s_{g \cdot e}^{*}\right)\left(s_{k \cdot f} s_{k \cdot f}^{*}\right) x=0
$$

thus $T_{G \cdot e}$ and $T_{G \cdot f}$ have othogonal ranges. A similar calculation shows that the projections $Q_{G \cdot v}$ have mutually orthogonal ranges, and that $\left(T_{G \cdot e} T_{G \cdot e}^{*}\right) Q_{G \cdot s(e)}=T_{G \cdot e} T_{G \cdot e}^{*}$, which implies the Cuntz-Krieger relation $T_{G \cdot e} T_{G \cdot e}^{*} \leq Q_{s(G \cdot e)}$. For the third CuntzKrieger relation, suppose $G \cdot v$ is a vertex in $G \backslash E$ such that $s^{-1}(G \cdot v)$ is finite and nonempty. Then

$$
s^{-1}(G \cdot v)=\left\{G \cdot e: e \in E^{1} \text { and } s(e)=v\right\},
$$

so we can use the Cuntz-Krieger relation $p_{v}=\sum_{s(e)=v} s_{e} s_{e}^{*}$ and (1.2) to see that

$$
Q_{G \cdot v}=I_{G}\left(p_{v}\right)=\sum_{s(e)=v} I_{G}\left(s_{e} s_{e}^{*}\right)=\sum_{s(e)=v} I_{G}\left(s_{e}\right) I_{G}\left(s_{e}\right)^{*}=\sum_{s(G \cdot e)=G \cdot v} T_{G \cdot e} T_{G \cdot e}^{*} .
$$

We take for $\phi_{G}$ the homomorphism $\pi_{T, Q}$ corresponding to this Cuntz-Krieger family; it is easy to see by looking at the projections $Q_{G \cdot v}$ that $\phi_{G}$ is nondegenerate. The last formula follows from (1.2). To see that $\phi_{G}$ is an isomorphism, note that each $Q_{G \cdot v}$ is nonzero and apply the gauge-invariant uniqueness theorem of [13, Theorem 2.7].

Corollary 1.5. The map $\phi_{G}$ of Lemma 1.4 is an isomorphism of $C^{*}(G \backslash E)$ onto the generalised fixed-point algebra $C^{*}(E)^{\alpha}$.

Proof. The generalised fixed-point algebra is by definition the closure in $M\left(C^{*}(E)\right)$ of

$$
\operatorname{span}\left\{I_{G}\left(x^{*} y\right): x, y \in X_{0}(E)\right\}=\operatorname{span}\left\{I_{G}\left(s_{\mu} s_{\nu}^{*}\right): \mu, \nu \in E^{*}\right\},
$$

which by (1.4) and (1.3) is the image of $\phi_{G}$. 
To prove that the action $\alpha$ is saturated, it suffices to show that the functions $g \mapsto x \alpha_{g}\left(y^{*}\right)$ span a dense ideal of the reduced crossed product $C^{*}(E) \times_{\alpha, r} G$. Indeed, because $G$ is discrete and the elements $s_{\mu} s_{\nu}^{*}$ span a dense subspace of $C^{*}(E)$, it suffices to show that the functions $f(g)=\delta_{k}(g) s_{\mu} s_{\nu}^{*}$ have this form. But

$$
f(g)=\delta_{k}(g) s_{\mu} s_{\nu}^{*} p_{s(\nu)}=s_{\mu} s_{\nu}^{*} \alpha_{g}\left(p_{k^{-1} \cdot s(\nu)}\right),
$$

so the span of the left inner-products $C^{*}(E) \times G\langle x, y\rangle$ is precisely the dense subalgebra $k\left(G, X_{0}(E)\right)$ of $C^{*}(E) \times_{r} G$. For later use, we record that the left action of $f=\delta_{k} s_{\mu} s_{\nu}^{*}$ is given by

$$
\begin{aligned}
f \cdot x & ={ }_{C^{*}(E) \times G}\left\langle s_{\mu} s_{\nu}^{*}, p_{k^{-1} \cdot s(\nu)}\right\rangle \cdot x:=\left(s_{\mu} s_{\nu}^{*}\right) \cdot\left\langle p_{k^{-1} \cdot s(\nu)}, x\right\rangle_{D} \\
& =\sum_{g}\left(s_{\mu} s_{\nu}^{*}\right) \alpha_{g}\left(p_{k^{-1} \cdot s(\nu)} x\right)=\sum_{g} f(g) \alpha_{g}(x),
\end{aligned}
$$

and this formula extends by linearity to all $f \in k\left(G, X_{0}(E)\right)$.

We can now deduce the following theorem from [16, Theorem 1.5].

Theorem 1.6. Suppose a group $G$ acts freely on a directed graph $E$, and let $\alpha$ be the induced action on $C^{*}(E)$. Then the reduced crossed product $C^{*}(E) \times_{\alpha, r} G$ is Morita equivalent to $C^{*}(G \backslash E)$.

We now consider the symmetric situation in which we have commuting free actions of groups $G$ and $H$ on the left and right of $E$. We continue to write $\alpha$ for the action of $G$ on $C^{*}(E)$ induced by the left action, and write $\beta$ for the corresponding action of $H$, which is characterised by $\beta_{h}\left(s_{e}\right)=s_{e \cdot h^{-1}}$. Because the action of $H$ commutes with that of $G$, it descends to an action on the quotient graph $G \backslash E$, and this induces an action on the $C^{*}$-algebra $C^{*}(G \backslash E)$. It should not cause too much confusion if we call this action $\beta$ too: see Lemma 1.7(3) below. In the same way, we write $\alpha$ for the induced action of $G$ on $C^{*}(E / H)$.

For the proof of our symmetric version of Theorem 1.6, we need two lemmas. The first describes how the actions interact with the averaging maps $I_{G}$ and $I_{H}$; by symmetry, it is enough to check one side.

Lemma 1.7. Let $x \in X_{0}(G \backslash E), y \in X_{0}(E)$ and $h \in H$. Then

(1) $\phi_{G}(x) I_{G}(y)=I_{G}\left(\phi_{G}(x) y\right)$;

(2) $\beta_{h}\left(I_{G}(y)\right)=I_{G}\left(\beta_{h}(y)\right)$;

(3) $\beta_{h}\left(\phi_{G}(x)\right)=\phi_{G}\left(\beta_{h}(x)\right)$.

Proof. For (1), just recall that $\phi_{G}(x)$ belongs to the fixed-point algebra $M\left(X_{0}(E)\right)^{\alpha}$, so for $z \in X_{0}(E)$ we have

$$
\left(\phi_{G}(x) I_{G}(y)\right) z=\phi_{G}(x)\left(\sum_{g \in G} \alpha_{g}(y) z\right)=\sum_{g \in G} \alpha_{g}\left(\phi_{G}(x) y\right) z=I_{G}\left(\phi_{G}(x) y\right) z .
$$

For (2), note that

$$
\beta_{h}\left(I_{G}(y)\right) z=\beta_{h}\left(I_{G}(y) \beta_{h}^{-1}(z)\right)=\beta_{h}\left(\sum_{g \in G} \alpha_{g}(y) \beta_{h}^{-1}(z)\right)=\sum_{g \in G} \beta_{h}\left(\alpha_{g}(y) \beta_{h}^{-1}(z)\right),
$$


and the result follows because $\beta_{h} \circ \alpha_{g}=\alpha_{g} \circ \beta_{h}$. Part (3) follows from (2) because $\phi_{G}\left(s_{G \cdot \mu} s_{G \cdot \nu}^{*}\right)$ is by definition $I_{G}\left(s_{\mu} s_{\nu}^{*}\right)$, and every $x$ is a linear combination of terms of the form $s_{G \cdot \mu} s_{G \cdot \nu}^{*}$.

The next lemma is standard; it is a very special case of [2, Theorem 2.1], for example.

Lemma 1.8. Suppose $\alpha$ and $\beta$ are commuting actions of $G$ and $H$ on a $C^{*}$-algebra $A$, and let $\alpha \times \beta$ denote the action of $G \times H$ on $A$ defined by $(\alpha \times \beta)_{(g, h)}=\alpha_{g} \circ \beta_{h}$. Then there is an action $\beta \times{ }_{r}$ id of $H$ on $A \times{ }_{\alpha, r} G$ such that $\left(\beta \times_{r} \mathrm{id}\right)_{h}(f)(s)=\beta_{h}(f(s))$ for $f \in k(G, A)$, and then

$$
\left(A \times_{\alpha, r} G\right) \times_{\beta \times_{r} \mathrm{id}, r} H \cong A \times_{\alpha \times \beta, r}(G \times H) .
$$

Theorem 1.9. Suppose that we have commuting free actions of two groups $G$ and $H$ on the left and right of a directed graph $E$, and let $\alpha: G \rightarrow C^{*}(E / H)$ and $\beta: H \rightarrow C^{*}(G \backslash E)$ denote the induced actions on the $C^{*}$-algebras of the quotient graphs. Then $C^{*}(G \backslash E) \times_{\beta, r} H$ is Morita equivalent to $C^{*}(E / H) \times_{\alpha, r} G$.

Proof. Define an action $u$ of $H$ on the bimodule $X=\bar{X}_{0}(E) \|^{\|\cdot\|_{D}}$ giving the equivalence between $C^{*}(E) \times_{\alpha, r} G$ and $C^{*}(E)^{\alpha}=\phi_{G}\left(C^{*}(G \backslash E)\right)$ by

$$
u_{h}\left(s_{\mu} s_{\nu}^{*}\right)=s_{\mu \cdot h^{-1}} s_{\nu \cdot h^{-1}}^{*} ;
$$

because the actions $\alpha$ and $\beta$ commute and $\beta$ pulls through the maps $I_{G}$ and $\phi_{G}, u_{h}$ is isometric for $\|\cdot\|_{D}$, and hence extends to the completion. The pair $(X, u)$ then implements a Morita equivalence between the systems $\left(C^{*}(E) \times_{\alpha, r} G, H, \beta \times_{r}\right.$ id $)$ and $\left(C^{*}(G \backslash E), H, \beta\right)$, and hence by [3, page 299] there is a Morita equivalence $X \times_{u, r} H$ between $\left(C^{*}(E) \times_{\alpha, r} G\right) \times_{\beta \times_{r} \text { id }, r} H$ and $C^{*}(G \backslash E) \times_{\beta, r} H$. Reversing the roles of $G$ and $H$ gives us an equivalence $Y \times_{v, r} G$ between $\left(C^{*}(E) \times_{\beta, r} H\right) \times_{\alpha \times_{r} \text { id, } r} G$ and $C^{*}(E / H) \times_{\alpha, r} G$. By Lemma 1.8, both iterated crossed products are isomorphic to $C^{*}(E) \times_{\alpha \times \beta, r}(G \times H)$, and now the internal tensor product

$$
\left(Y \times_{u, r} H\right)^{\sim} \otimes_{C^{*}(E) \times_{\alpha \times \beta, r}(G \times H)}\left(X \times_{v, r} G\right)
$$

implements the required equivalence (see $[14, \S 3.2]$ ).

\section{Full CROSSEd PROduCTS}

Theorem 2.1. Suppose that we have commuting free actions of two groups $G$ and $H$ on the left and right of a directed graph $E$, and let $\alpha: G \rightarrow C^{*}(E / H)$ and $\beta: H \rightarrow C^{*}(G \backslash E)$ denote the induced actions on the $C^{*}$-algebras of the quotient graphs. Then $C^{*}(G \backslash E) \times{ }_{\beta} H$ is Morita equivalent to $C^{*}(E / H) \times{ }_{\alpha} G$.

We shall prove this theorem by making

$$
X_{0}(E):=\overline{\operatorname{span}}\left\{s_{\mu} s_{\nu}^{*}: \mu, \nu \in E^{*}\right\}
$$

into a pre-imprimitivity bimodule over the dense subalgebras $B_{0}:=k\left(H, X_{0}(G \backslash E)\right)$ of $B:=C^{*}(G \backslash E) \times_{\beta} H$ and $C_{0}:=k\left(G, X_{0}(E / H)\right)$ of $C:=C^{*}(E / H) \times_{\alpha} G$, as in 
[14, Definition 3.9]. For $b \in B_{0}, c \in C_{0}$ and $x, y \in X_{0}(E)$, define

$$
\begin{gathered}
b \cdot x=\sum_{h \in H} \phi_{G}(b(h)) \beta_{h}(x) \text { and } x \cdot c=\sum_{g \in G} \alpha_{g}^{-1}\left(x \phi_{H}(c(g))\right), \\
{ }_{B}\langle x, y\rangle(h)=\phi_{G}^{-1} \circ I_{G}\left(x \beta_{h}\left(y^{*}\right)\right) \text { and }\langle x, y\rangle_{C}(g)=\phi_{H}^{-1} \circ I_{H}\left(x^{*} \alpha_{g}(y)\right) .
\end{gathered}
$$

Before starting to verify that these actions and inner products have the right properties, it pays to observe that the situation is more symmetric than it appears at first sight. Indeed, as in [12, page 372], the map $\Phi: x \mapsto x^{*}$ on $X_{0}(E)$ obviously swaps the inner products, and converts the formula for the left action into the one for the right action:

$$
\begin{aligned}
\Phi(b \cdot x) & =\sum_{h \in H} \beta_{h}\left(x^{*}\right) \phi_{G}\left(b(h)^{*}\right)=\sum_{h \in H} \beta_{h}\left(\Phi(x) \phi_{G}\left(b^{*}\left(h^{-1}\right)\right)\right) \\
& =\sum_{h \in H} \beta_{h}^{-1}\left(\Phi(x) \phi_{G}\left(b^{*}(h)\right)\right) .
\end{aligned}
$$

So it is enough to check many of the properties on just one side.

We begin by checking the algebraic properties.

Lemma 2.2. Let $b_{1}, b_{2} \in k\left(H, X_{0}(G \backslash E)\right)$ and $x, y \in X_{0}(E)$. Then

$$
b_{1} \cdot\left(b_{2} \cdot x\right)=\left(b_{1} b_{2}\right) \cdot x, \quad{ }_{B}\left\langle b_{1} \cdot x, y\right\rangle=b_{1} \cdot{ }_{B}\langle x, y\rangle \quad \text { and } \quad\left({ }_{B}\langle x, y\rangle\right)^{*}={ }_{B}\langle y, x\rangle .
$$

Proof. We compute:

$$
\begin{aligned}
b_{1} \cdot\left(b_{2} \cdot x\right) & =\sum_{k \in H} \phi_{G}\left(b_{1}(k)\right) \beta_{k}\left(\sum_{h \in H} \phi_{G}\left(b_{2}(h)\right) \beta_{h}(x)\right) \\
& =\sum_{k \in H} \phi_{G}\left(b_{1}(k)\right)\left(\sum_{h \in H} \phi_{G}\left(\beta_{k} b_{2}(h)\right) \beta_{k h}(x)\right) \quad \text { by Lemma } 1.7 \\
& =\sum_{k \in H} \sum_{\ell \in H} \phi_{G}\left(b_{1}(k) \beta_{k}\left(b_{2}\left(k^{-1} \ell\right)\right)\right) \beta_{\ell}(x) \quad \text { putting } \ell=k h \\
& =\sum_{\ell \in H} \phi_{G}\left(b_{1} b_{2}(\ell)\right) \beta_{\ell}(x) \\
& =\left(b_{1} b_{2}\right) \cdot x
\end{aligned}
$$


And again:

$$
\begin{aligned}
{ }_{B}\langle b \cdot x, y\rangle(h) & =\phi_{G}^{-1} \circ I_{G}\left(\sum_{k \in H} \phi_{G}(b(k)) \beta_{k}(x) \beta_{h}\left(y^{*}\right)\right) \\
& =\phi_{G}^{-1}\left(\sum_{k \in H} \phi_{G}(b(k)) I_{G}\left(\beta_{k}(x) \beta_{h}\left(y^{*}\right)\right)\right) \quad \text { by Lemma } 1.7(1) \\
& =\sum_{k \in H} b(k) \phi_{G}^{-1} \circ I_{G}\left(\beta_{k}\left(x \beta_{k^{-1} h}\left(y^{*}\right)\right)\right) \\
& =\sum_{k \in H} b(k) \beta_{k}\left(\phi_{G}^{-1} \circ I_{G}\left(x \beta_{k^{-1} h}\left(y^{*}\right)\right)\right) \text { by Lemma } 1.7 \\
& =\left(b \cdot{ }_{B}\langle x, y\rangle\right)(h) .
\end{aligned}
$$

The last statement follows from more calculations using Lemma 1.7.

To establish the positivity of the inner products, we need to know how the left and right structures interact.

Lemma 2.3. For $b \in B_{0}, c \in C_{0}$ and $x, y, z \in X_{0}(E)$, we have

$$
(b \cdot x) \cdot c=b \cdot(x \cdot c), \quad \text { and } \quad x \cdot\langle y, z\rangle_{C}={ }_{B}\langle x, y\rangle \cdot z .
$$

Proof. Once again, we compute using Lemma 1.7:

$$
\begin{aligned}
(b \cdot x) \cdot c & =\sum_{g \in G} \sum_{h \in H} \alpha_{g}^{-1}\left(\phi_{G}(b(h)) \beta_{h}(x) \phi_{H}(c(g))\right) \\
& =\sum_{h \in H} \sum_{g \in G} \phi_{G}(b(h)) \beta_{h}\left(\alpha_{g}^{-1}\left(x \phi_{H}(c(g))\right)\right) \\
& =b \cdot(x \cdot c) .
\end{aligned}
$$

The second equation follows almost immediately from the characterising properties (1.1) of $I_{G}$ and $I_{H}$.

Lemma 2.4. For every $x \in X_{0}(E),{ }_{B}\langle x, x\rangle$ is a positive element of the $C^{*}$-algebra completion $B=C^{*}(G \backslash E) \times_{\beta} H$ of $B_{0}$, and $X_{0}(E)$ is a left pre-inner-product $B_{0}$ module. Similarly, $X_{0}(E)$ is a right pre-inner-product $C_{0}$-module.

Proof. In view of Lemma 2.2 and the symmetry of the situation, it suffices to prove the first statement. Recall that $x$ is a finite sum $\sum_{\mu, \nu} \lambda_{\mu, \nu} s_{\mu} s_{\nu}^{*}$; let $F$ be a set of representatives in $E^{0}$ for

$$
\left\{v \cdot H \in(E / H)^{0}: v=s(\nu) \text { for some }(\mu, \nu) \text { with } \lambda_{\mu, \nu} \neq 0\right\} .
$$

Observe that

$$
\sum_{v \in F}\left\langle p_{v}, p_{v}\right\rangle_{C}(g)= \begin{cases}\sum_{v \in F} \phi_{H}^{-1} \circ I_{H}\left(p_{v}\right) & \text { if } g=e_{G} \\ 0 & \text { otherwise }\end{cases}
$$


so the choice of $F$ implies that

$$
x \cdot \sum_{v \in F}\left\langle p_{v}, p_{v}\right\rangle_{C}=\sum_{v \in F} x I_{H}\left(p_{v}\right)=\sum_{v \in F} \sum_{h \in H} x p_{v \cdot h}=x .
$$

Thus from Lemma 2.3 we have

$$
\begin{aligned}
{ }_{B}\langle x, x\rangle & ={ }_{B}\left\langle x, x \cdot\left(\sum_{v \in F}\left\langle p_{v}, p_{v}\right\rangle_{C}\right)\right\rangle=\sum_{v \in F} B\left\langle x, x \cdot\left\langle p_{v}, p_{v}\right\rangle_{C}\right\rangle \\
& =\sum_{v \in F}{ }_{B}\left\langle x,{ }_{B}\left\langle x, p_{v}\right\rangle \cdot p_{v}\right\rangle=\sum_{v \in F}\left({ }_{B}\left\langle x, p_{v}\right\rangle\right)^{*}{ }_{B}\left\langle x, p_{v}\right\rangle,
\end{aligned}
$$

which is positive in $B$.

In view of Lemma 2.4 and Lemma 2.3, it remains to establish property (c) of [14, Definition 3.9], namely that

$$
\|b\|^{2}\langle x, x\rangle_{C}-\langle b \cdot x, b \cdot x\rangle_{C} \geq 0 \text { in } C .
$$

To do this, we choose a faithful representation $\pi$ of $C=\overline{C_{0}}$ on $\mathcal{H}_{\pi}$. Since we know that $X_{0}(E)$ is a pre-inner-product $C_{0}$-module, we can induce to get a faithful representation of $\mathcal{L}\left(X_{0}(E)\right)$ on $\mathcal{H}:=\overline{X_{0}(E) \otimes_{C} \mathcal{H}_{\pi}}$. We shall construct a covariant representation $(\rho, U)$ of $\left(C^{*}(G \backslash E), H, \beta\right)$ on $\mathcal{H}$ such that

$$
\rho \times U(b)(x \otimes \xi)=(b \cdot x) \otimes \xi \text { for } b \in B_{0} \text { and } x \in X_{0}(E) .
$$

Then, because every representation of a $C^{*}$-algebra is norm-decreasing, we can deduce that

$$
\|(b \cdot x) \otimes \xi\|^{2}=\|\rho \times U(b)(x \otimes \xi)\|^{2} \leq\|b\|^{2}\|x \otimes \xi\|^{2} \text { for every } \xi \in \mathcal{H}_{\pi},
$$

or, equivalently,

$$
0 \leq\left(\pi\left(\|b\|^{2}\langle x, x\rangle_{C}-\langle b \cdot x, b \cdot x\rangle_{C}\right) \xi \mid \xi\right) \text { for every } \xi \in \mathcal{H}_{\pi} .
$$

Thus $\pi\left(\|b\|^{2}\langle x, x\rangle_{C}-\langle b \cdot x, b \cdot x\rangle_{C}\right)$ is a positive operator on $\mathcal{H}_{\pi}$, which implies (2.3) because $\pi$ is faithful.

To construct the first part $\rho$ of our covariant pair $(\rho, U)$, we show that there is a Cuntz-Krieger $(G \backslash E)$-family $\left\{S_{G \cdot e}, P_{G \cdot v}\right\}$ on $\mathcal{H}$ such that

$$
S_{G \cdot e}(x \otimes \xi)=\left(I_{G}\left(s_{e}\right) x\right) \otimes \xi \text { and } P_{G \cdot v}(x \otimes \xi)=\left(I_{G}\left(p_{v}\right) x\right) \otimes \xi
$$

for $x \otimes \xi \in X_{0}(E) \odot \mathcal{H}_{\pi}$. We take $P_{G \cdot v}$ to be the orthogonal projection on

$$
\overline{\operatorname{span}}\left\{s_{\mu} s_{\nu}^{*} \otimes \xi: s(\mu) \in G \cdot v\right\} \text {; }
$$

since

$$
\left(s_{\mu} s_{\nu}^{*} \otimes \xi \mid s_{\alpha} s_{\beta}^{*} \otimes \eta\right)=\left(\pi\left(g \mapsto \phi_{H}^{-1} \circ I_{H}\left(s_{\nu} s_{\mu}^{*} s_{g \cdot \alpha} s_{g \cdot \beta}^{*}\right)\right) \xi \mid \eta\right)
$$

vanishes unless $G \cdot s(\mu)=G \cdot s(\alpha)$, the $P_{G \cdot v}$ have mutually orthogonal ranges spanning $\mathcal{H}$.

To see that the formula for $S_{G \cdot e}$ defines a partial isometry, we first deduce from the relation $I_{G}\left(s_{g}\right)^{*} I_{G}\left(s_{g}\right)=I_{G}\left(p_{r(e)}\right)$ established in Lemma 1.4 that $S_{G \cdot e}=S_{G \cdot e} P_{G \cdot r(e)}$; thus $S_{G \cdot e}$ vanishes on the complement of $P_{G \cdot r(e)} \mathcal{H}$, and it suffices to show that $S_{G \cdot e}$ 
is isometric on $P_{G \cdot r(e)} \mathcal{H}$. If $G \cdot s(\mu)=G \cdot s(\alpha)=G \cdot r(e)$, then there are unique elements $g_{\mu}, g_{\alpha} \in G$ such that $s(\mu)=g_{\mu} \cdot r(e)$ and $s(\alpha)=g_{\alpha} \cdot r(e)$, and

$$
\begin{aligned}
\left\langle I_{G}\left(s_{e}\right)\left(s_{\alpha} s_{\beta}^{*}\right), I_{G}\left(s_{e}\right)\right. & \left.\left(s_{\mu} s_{\nu}^{*}\right)\right\rangle_{C}(g)=\left\langle s_{g_{\alpha} \cdot e} s_{\alpha} s_{\beta}^{*}, s_{g_{\mu} \cdot e} s_{\mu} s_{\nu}^{*}\right\rangle_{C}(g) \\
& =\phi_{H}^{-1} \circ I_{H}\left(s_{\beta} s_{\alpha}^{*} s_{g_{\alpha} \cdot e}^{*} s_{\left(g g_{\mu}\right) \cdot e} s_{g \cdot \mu} s_{g \cdot \nu}^{*}\right) \\
& = \begin{cases}\phi_{H}^{-1} \circ I_{H}\left(s_{\beta} s_{\alpha}^{*} s_{g \cdot \mu} s_{g \cdot \nu}^{*}\right) & \text { if } g g_{\mu} \cdot r(e)=g_{\alpha} \cdot r(e) \\
0 & \text { otherwise. }\end{cases}
\end{aligned}
$$

Because $G$ acts freely, this vanishes for all $g$ except $g=g_{\alpha} g_{\mu}^{-1}$, which is the only $g$ for which $g \cdot s(\mu)=s(\alpha)$; thus (2.5) reduces to $\left\langle s_{\alpha} s_{\beta}^{*}, s_{\mu} s_{\nu}^{*}\right\rangle_{C}(g)$. Now we can compute

$$
\begin{aligned}
\left(S_{G \cdot e}\left(s_{\mu} s_{\nu}^{*} \otimes \eta\right) \mid S_{G \cdot e}\right. & \left.\left(s_{\alpha} s_{\beta}^{*} \otimes \xi\right)\right)=\left(I_{G}\left(s_{e}\right)\left(s_{\mu} s_{\nu}^{*}\right) \otimes \eta \mid I_{G}\left(s_{e}\right)\left(s_{\alpha} s_{\beta}^{*}\right) \otimes \xi\right) \\
& =\left(\pi\left(\left\langle s_{\alpha} s_{\beta}^{*}, s_{\mu} s_{\nu}^{*}\right\rangle_{C}\right) \eta \mid \xi\right) \text { by the previous calculation } \\
& =\left(s_{\mu} s_{\nu}^{*} \otimes \zeta \mid s_{\alpha} s_{\beta}^{*} \otimes \xi\right) .
\end{aligned}
$$

It follows easily that $S_{G \cdot e}$ is isometric on $\operatorname{span}\left\{s_{\mu} s_{\nu}^{*}: s(\mu) \in G \cdot r(e)\right\}$, and hence extends to a partial isometry, as claimed.

We next claim that the adjoint of $S_{G \cdot e}$ in $B(\mathcal{H})$ is given on $X_{0}(E) \otimes \mathcal{H}_{\pi}$ by

$$
S_{G \cdot e}(x \otimes \xi)=\left(I_{G}\left(s_{e}^{*}\right) x\right) \otimes \xi=\left(I_{G}\left(s_{e}\right)^{*} x\right) \otimes \xi .
$$

(This will allow us to use the Cuntz-Krieger $G \backslash E$-relations established in Lemma 1.4.) To see (2.6), it suffices to show that

$$
\left\langle s_{\gamma} s_{\delta}^{*}, I_{G}\left(s_{e}^{*}\right) s_{\mu} s_{\nu}^{*}\right\rangle_{C}(g)=\left\langle I_{G}\left(s_{e}\right) s_{\gamma} s_{\delta}^{*}, s_{\mu} s_{\nu}^{*}\right\rangle_{C}(g)
$$

for all $g \in G$, which will follow from

$$
s_{\delta} s_{\gamma}^{*} \alpha_{g}\left(I_{G}\left(s_{e}^{*}\right) s_{\mu} s_{\nu}^{*}\right)=\left(I_{G}\left(s_{e}\right) s_{\gamma} s_{\delta}^{*}\right)^{*} \alpha_{g}\left(s_{\mu} s_{\nu}^{*}\right) .
$$

We look at the left-hand side of (2.7) first. The product $I_{G}\left(s_{e}^{*}\right) s_{\mu}$ vanishes unless $G \cdot e=G \cdot \mu_{1}$, and then $I_{G}\left(s_{e}^{*}\right) s_{\mu}=s_{\mu_{2}} \cdots s_{\mu_{|\mu|}}=s_{\mu^{\prime}}$, say. Thus

$$
s_{\delta} s_{\gamma}^{*} \alpha_{g}\left(I_{G}\left(s_{e}^{*}\right) s_{\mu} s_{\nu}^{*}\right)= \begin{cases}s_{\delta} s_{\gamma}^{*} s_{g \cdot \mu^{\prime}} s_{g \cdot \nu} & \text { if } G \cdot e=G \cdot \mu_{1} \text { and } g \cdot s\left(\mu_{2}\right)=s(\gamma), \\ 0 & \text { otherwise. }\end{cases}
$$

Now we consider the right-hand side of (2.7). The product $I_{G}\left(s_{e}\right) s_{\gamma}$ vanishes unless $G \cdot r(e)=G \cdot s(\gamma)$, and then $I_{G}\left(s_{e}\right) s_{\gamma}=s_{g_{\gamma} \cdot e} s_{\gamma}$ for the unique element $g_{\gamma}$ of $G$ such that $g_{\gamma} \cdot r(e)=s(\gamma)$. Now $s_{g_{\gamma} \cdot e}^{*} s_{g \cdot \mu}$ vanishes unless $g_{\gamma} \cdot e=g \cdot \mu_{1}$, and then equals $s_{g \cdot \mu^{\prime}}$. Thus

$$
\left(I_{G}\left(s_{e}\right) s_{\gamma} s_{\delta}^{*}\right)^{*} \alpha_{g}\left(s_{\mu} s_{\nu}^{*}\right)= \begin{cases}s_{\delta} s_{\gamma}^{*} s_{g \cdot \mu^{\prime}} s_{g \cdot \nu} & \text { if } G \cdot r(e)=G \cdot s(\gamma) \text { and } g_{\gamma} \cdot e=g \cdot \mu_{1}, \\ 0 & \text { otherwise. }\end{cases}
$$

Now we have to check that the two sets of constraints on $g$ are equivalent. For one direction, $G \cdot e=G \cdot \mu_{1}$ implies that there exists $g^{\prime}$ with $g^{\prime} \cdot e=\mu_{1}$, and then $g \cdot s\left(\mu_{2}\right)=s(\gamma)$ implies $s(\gamma)=s\left(g \cdot \mu_{2}\right)=r\left(g \cdot \mu_{1}\right)=r\left(g g^{\prime} \cdot e\right)$, so $G \cdot r(e)=G \cdot s(\gamma)$ 
with $g_{\gamma}=g g^{\prime}$. For the other direction, note that $g_{\gamma} \cdot e=g \cdot \mu_{1}$ certainly implies $G \cdot e=G \cdot \mu_{1}$, and then $g \cdot s\left(\mu_{2}\right)=r\left(g \cdot \mu_{1}\right)=r\left(g_{\gamma} \cdot e\right)=s(\gamma)$ by choice of $g_{\gamma}$. We have now proved (2.7), and hence also (2.6).

Since we know from Lemma 1.4 that $\left\{I_{G}\left(s_{e}\right), I_{G}\left(p_{v}\right)\right\}$ is a Cuntz-Krieger $(G \backslash E)$ family in $M\left(X_{0}(E)\right)$, we can deduce from calculations on elementary tensors in $X_{0}(E) \otimes \mathcal{H}_{\pi}$ that $\left\{S_{G \cdot e}, P_{G \cdot v}\right\}$ is a Cuntz-Krieger $(G \backslash E)$-family on $\mathcal{H}$. For example, if $s^{-1}(G \cdot v)$ is finite and nonempty, we have

$$
\begin{aligned}
\sum_{s(G \cdot e)=G \cdot v} S_{G \cdot e} S_{G \cdot e}^{*}(x \otimes \xi) & =\left(\sum_{s(G \cdot e)=G \cdot v} I_{G}\left(s_{e}\right) I_{G}\left(s_{e}\right)^{*} x\right) \otimes \xi \\
& =\left(I_{G}\left(p_{v}\right) x\right) \otimes \xi=P_{G \cdot v}(x \otimes \xi) .
\end{aligned}
$$

We can now define the first part of the covariant representation $(\rho, U)$ which we seek by taking $\rho:=\pi_{S, P}: C^{*}(G \backslash E) \rightarrow B(\mathcal{H})$. We define $U: H \rightarrow U(\mathcal{H})$ by $U_{h}(x \otimes \xi)=\beta_{h}(x) \otimes \xi$. Each $U_{h}$ is unitary because it is invertible (with $\left(U_{h}\right)^{-1}=$ $\left.U_{h^{-1}}\right)$ and isometric:

$$
\begin{aligned}
\left\langle\beta_{h}(x), \beta_{h}(y)\right\rangle_{C}(g) & =\phi_{H}^{-1} \circ I_{H}\left(\beta_{h}(x)^{*} \alpha_{g}\left(\beta_{h}(y)\right)\right)=\phi_{H}^{-1} \circ I_{H}\left(\beta_{h}\left(x^{*} \alpha_{g}(y)\right)\right) \\
& =\phi_{H}^{-1} \circ I_{H}\left(x^{*} \alpha_{g}(y)\right)=\langle x, y\rangle_{C}(g) .
\end{aligned}
$$

Covariance follows from the identity $\beta_{h}\left(I_{G}(x)\right)=I_{G}\left(\beta_{h}(x)\right)$ :

$$
\begin{aligned}
U_{h} \rho\left(\beta_{h}\left(s_{G \cdot e}\right)\right)(x \otimes \xi) & =U_{h} \rho\left(s_{G \cdot\left(e \cdot h^{-1}\right)}\right)(x \otimes \xi)=\beta_{h}\left(I_{G}\left(s_{e \cdot h^{-1}}\right) x\right) \otimes \xi \\
& =\left(I_{G}\left(\beta_{h}\left(s_{e \cdot h^{-1}}\right)\right) \beta_{h}(x)\right) \otimes \xi=\left(I_{G}\left(s_{e}\right) \beta_{h}(x)\right) \otimes \xi \\
& =\rho\left(s_{G \cdot e}\right) U_{h}(x \otimes \xi) .
\end{aligned}
$$

Finally, we need to check that $\rho \times U(b)$ satisfies (2.4). But since every path in $G \backslash E$ has the form $G \cdot \mu$ for some path $\mu$ in $E$, it suffices to note that $\rho\left(s_{G \cdot \mu} s_{G \cdot \nu}^{*}\right)=$ $I_{G}\left(s_{\mu} s_{\nu}^{*}\right)=\phi_{G}\left(s_{G \cdot \mu} s_{G \cdot \nu}^{*}\right)$, and that the action of $B_{0}$ on $X_{0}(E)$ is formally the integrated form of $\left(\phi_{G}, \beta\right)$ (see (2.1)).

This completes the construction of $(\rho, U)$, and hence the verification of [14, Definition $3.9(\mathrm{c})$ ]. We have now shown that $X_{0}(E)$ is a $B_{0}-C_{0}$ pre-imprimitivity bimodule in the sense of [14, Definition 3.9], and it follows from [14, Proposition 3.12] that the completion is a $\left(C^{*}(G \backslash E) \times H\right)-\left(C^{*}(E / H) \times G\right)$ imprimitivity bimodule.

This completes the proof of Theorem 2.1.

\section{Applications}

3.1. Amenability. We now show how comparing the two bimodules we have constructed can give information about amenability of the actions. The idea for this comes from [8].

Corollary 3.1 ([9, Corollary 3.3]). Suppose $G$ acts freely on a directed graph $E$, and let $\alpha$ denote the induced action of $G$ on $C^{*}(E)$. Then $\alpha$ is amenable in the sense that $C^{*}(E) \times_{\alpha} G=C^{*}(E) \times_{\alpha, r} G$. 
Proof. Define a right action of $G$ on $E$ by $e \cdot g:=g^{-1} \cdot e$, and apply Theorem 2.1 with the other group absent. This gives a $\left(C^{*}(E) \times G\right)-C^{*}(G / E)$ imprimitivity module $\overline{X_{0}(E)}$ in which the norm comes from the $C^{*}(G \backslash E)$-valued inner product. Since $\phi_{G}$ is isometric, for $x \in X_{0}(E)$ we have

$$
\|x\|^{2}=\left\|\langle x, x\rangle_{C^{*}(G \backslash E)}\right\|=\left\|\phi_{G}^{-1} \circ I_{G}\left(x^{*} x\right)\right\|=\left\|I_{G}\left(x^{*} x\right)\right\|=\left\|\langle x, x\rangle_{D}\right\| .
$$

Thus the completions of $X_{0}(E)$ in Theorems 1.6 and 2.1 are the same as Hilbert $C^{*}(G \backslash E)$-modules, and have the same left action of $B_{0}$ (compare (1.5) with (2.1)). In particular, this implies that the ideal in $C^{*}(G \backslash E)$ associated by the Rieffel correspondence to the kernel of the regular representation of $C^{*}(E) \times G$ is $\{0\}$, and hence the regular representation is itself faithful.

Remark 3.2. It seems likely that a quotient of the bimodule of Theorem 2.1 will implement a Morita equivalence between $C^{*}(G \backslash E) \times_{\beta, r} H$ and $C^{*}(E / H) \times_{\alpha, r} G$, and hence that $\alpha$ is amenable exactly when $\beta$ is. However, as it stands it is not easy to compare the bimodule in Theorem 2.1 with the tensor product module constructed in the proof of Theorem 1.9.

In the usual symmetric imprimitivity theorem for free and proper actions on a space ${ }_{G} P_{H}$, the quotient spaces $G \backslash P$ and $P / H$ are always nice, but the actions on them need not be. In the same spirit, the actions $\alpha$ and $\beta$ will not always be amenable on $C^{*}(G \backslash E)$ and $C^{*}(E / H)$. For a somewhat trivial example, take $G$ to be a nonamenable group, and let $E$ be the graph with vertex set $G$ and no edges, so that $C^{*}(E)=c_{0}(G)$. If now $H=G$ also, then $C^{*}(E / H)=c_{0}(G / G)=\mathbb{C}$, and $C^{*}(E / H) \times G \cong C^{*}(G) \neq C_{r}^{*}(G)=C^{*}(E / H) \times{ }_{r} G$.

3.2. Induced actions. We start with a group $G$, a subgroup $H$ of $G$, and a right action of $H$ on a graph $E$. We consider the product graph $G \times E$ in which $r(g, e)=$ $(g, r(e))$ and $s(g, e)=(g, s(e))$, and define a right action of $H$ on $G \times E$ by

$$
(g, v) \cdot h=(g h, v \cdot h), \quad(g, e) \cdot h=(g h, e \cdot h) .
$$

This action is free because $H$ acts freely on $G$ in the first variable, and it commutes with the free left action of $G$ defined by $g_{1} \cdot(g, e)=\left(g_{1} g, e\right)$. We can therefore apply Theorem 2.1. The map $(g, e) \mapsto e$ induces an $H$-equivariant isomorphism of $G \backslash(G \times E)$ onto $E$, and hence an isomorphism of $C^{*}(G \backslash(G \times E)) \times{ }_{\beta} H$ onto the crossed product $C^{*}(E) \times_{\gamma} H$ by the action $\gamma$ induced by the original action of $H$. Thus, without any hypotheses on the action of $H$, we have:

Corollary 3.3. In the above notation, $C^{*}((G \times E) / H) \times_{\alpha} G$ is Morita equivalent to $C^{*}(E) \times_{\gamma} H$.

We can think of the action of $G$ on the graph $(G \times E) / H$ as being induced from the action of the subgroup $H$ on $E$, much as we induce group representations or actions on $C^{*}$-algebras. When we think this way it is natural to wonder how the graph-theoretic construction relates to the $C^{*}$-algebraic one, and, remarkably, it turns out to be the same, in the sense that $\left(C^{*}((G \times E) / H), G, \alpha\right)$ is isomorphic to $\left(\operatorname{Ind}_{H}^{G}\left(C^{*}(E), \gamma\right), G, \tau\right)$, where $\tau$ denotes the induced action by left translation. 
To see this, recall that if $H$ is a closed subgroup of a locally compact group and $\gamma: H \rightarrow$ Aut $A$, the induced $C^{*}$-algebra $\operatorname{Ind}_{H}^{G}(A, \gamma)$ is the subalgebra of $C_{b}(G, A)$ consisting of the functions $f$ satisfying $f(g h)=\gamma_{h}^{-1}(f(g))$ for $h \in H$ and such that $g H \mapsto\|f(g)\|$ belongs to $C_{0}(G / H)$. If $z \in C_{c}(G)$ and $a \in A$, then

$$
(z \odot a)(g):=\int_{H} f(g h) \gamma_{h}(a) d h
$$

defines an element $z \odot a$ of $\operatorname{Ind}_{H}^{G}(A, \gamma)$, and a partition of unity argument in $G / H$ shows that these elements span a dense subspace of $\operatorname{Ind}_{H}^{G}(A, \gamma)$. In our situation, everything is discrete, so the elements $\chi_{\{g\}} \odot s_{\mu} s_{\nu}^{*}$ span a dense subspace of $\operatorname{Ind}_{H}^{G}\left(C^{*}(E), \gamma\right)$. We can see by writing down a Cuntz-Krieger $(G \times E)$-family in $c_{0}(G) \otimes C^{*}(E)$ and applying the gauge-invariant uniqueness theorem of $[13$, Theorem 2.7] that $C^{*}(G \times E)$ is naturally isomorphic to $c_{0}(G) \otimes C^{*}(E)$. If we use this isomorphism to identify $C^{*}(G \times E)$ with $c_{0}(G) \otimes C^{*}(E)$, then, in our notation, $\chi_{\{g\}} \odot s_{\mu} s_{\nu}^{*}$ is just $I_{H}\left(\chi_{\{g\}} \odot s_{\mu} s_{\nu}^{*}\right)$. Thus $C^{*}(G \times E)^{\beta}$ is the subalgebra $\operatorname{Ind}_{H}^{G}\left(C^{*}(E), \gamma\right)$ of $C_{b}\left(G, C^{*}(E)\right) \subset M\left(C^{*}(G \times E)\right)$, and $\phi_{H}$ is an isomorphism of $C^{*}((G \times E) / H)$ onto $\operatorname{Ind}_{H}^{G}\left(C^{*}(E), \gamma\right)$. Since both $\alpha$ and $\tau$ act only on the copy of $G$, this isomorphism is equivariant, as claimed.

It is curious that we can now recover Corollary 3.3 from the usual symmetric imprimitivity theorem, which implies that

$$
C^{*}((G \times E) / H) \times_{\alpha} G \cong\left(\operatorname{Ind}_{H}^{G}\left(C^{*}(E), \gamma\right)\right) \times_{\tau} G
$$

is Morita equivalent to $C^{*}(E) \times_{\gamma} H$ (see $[12, \S 1.5]$ ). It is not at all clear to us, however, how one might try to recover the full strength of Theorem 2.1 from [12].

3.3. Green's imprimitivity theorem. Suppose a group $G$ acts on the left of a directed graph $E$, and consider the diagonal left action on $G \times E$. A subgroup $H$ of $G$ acts on the right of $G \times E$ by $(g, e) \cdot h=(g h, e)$, and we then have $(G \times E) / H \cong$ $(G / H) \times E$, equivariantly for the diagonal actions of $G$. The map $(g, e) \mapsto g^{-1} \cdot e$ induces an isomorphism of the other quotient $G \backslash(G \times E)$ onto $E$, which converts the right action of $H$ into that given in terms of the $G$-action by $e \cdot h:=h^{-1} \cdot e$. The action of $H$ on $C^{*}(E)$ induced by this right action is just the restriction of the original action $\alpha$ of $G$ on $C^{*}(E)$. On identifying $C^{*}((G / H) \times E)$ with $c_{0}(G / H) \otimes C^{*}(E)$, we recover:

Corollary 3.4. The crossed product $\left(c_{0}(G / H) \otimes C^{*}(E)\right) \times_{\tau \otimes \alpha} G$ is Morita equivalent to $C^{*}(E) \times{ }_{\alpha} H$.

This is precisely Green's imprimitivity theorem for the action $\alpha: G \rightarrow \operatorname{Aut} C^{*}(E)$ $([7, \S 2]$; see also $[12, \S 1.4])$.

\section{REFERENCES}

[1] T. Bates, D. Pask, I. Raeburn and W. Szymański, The $C^{*}$-algebras of row-finite graphs, preprint, U. of Newcastle, February 1999. 
[2] E. Bédos, Discrete groups and simple $C^{*}$-algebras, Math. Proc. Camb. Phil. Soc. 109 (1991), 521-537.

[3] F. Combes, Crossed products and Morita equivalence, Proc. London Math. Soc. 49 (1984), 289-306.

[4] R.E. Curto, P.S. Muhly and D.P. Williams, Cross products of strongly Morita equivalent $C^{*}$ algebras, Proc. Amer. Math. Soc. 90 (1984), 528-530.

[5] R. Exel, Morita-Rieffel equivalence and spectral theory for integrable automorphism groups of $C^{*}$-algebras, J. Funct. Anal. 172 (2000), 404-465.

[6] P. Green, $C^{*}$-algebras of transformation groups with smooth orbit space, Pacific J. Math. 72 (1977), 71-97.

[7] P. Green, The local structure of twisted covariance algebras, Acta Math. 140 (1978), 191-250.

[8] A. an Huef, I. Raeburn and D.P. Williams, An equivariant Brauer semigroup and the symmetric imprimitivity theorem, Trans. Amer. Math. Soc. 352 (2000), 4759-4787.

[9] S. Kaliszewski, J. Quigg and I. Raeburn, Skew products and crossed products by coactions, J. Operator Theory, to appear.

[10] A. Kumjian and D. Pask, $C^{*}$-algebras of directed graphs and group actions, Ergodic Th. \& Dynam. Sys. 19 (1999), 1503-1519.

[11] A. Kumjian, D. Pask and I. Raeburn, Cuntz-Krieger algebras of directed graphs, Pacific J. Math. 184 (1998), 161-174.

[12] I. Raeburn, Induced $C^{*}$-algebras and a symmetric imprimitivity theorem, Math. Ann. 280 (1988), 369-387.

[13] I. Raeburn and W. Szymański, Cuntz-Krieger algebras of infinite graphs and matrices, preprint, U. of Newcastle, January 2000.

[14] I. Raeburn and D.P. Williams, Morita Equivalence and Continuous-Trace $C^{*}$-Algebras, Math. Surveys \& Monographs, vol. 60, Amer. Math. Soc., Providence, 1998.

[15] M.A. Rieffel, Applications of strong Morita equivalence to transformation group algebras, Operator Algebras and Applications (R.V. Kadison, ed.), Proc. Sympos. Pure Math., vol. 38, Part I, Amer. Math. Soc., Providence, 1982, pages 299-310.

[16] M.A. Rieffel, Proper actions of groups on $C^{*}$-algebras, Mappings of Operator Algebras (H. Araki and R.V. Kadison, eds.), Progress in Math., vol. 84, Birkhäuser, Boston, 1989, pages $613-637$.

[17] M.A. Rieffel, Integrable and proper actions on $C^{*}$-algebras, and square integrable representations of groups, preprint, U. of California, Berkeley, June 1999.

Department of Mathematics, University of Newcastle, NSW 2308, Australia

E-mail address: davidp and iain@frey.newcastle.edu.au 